\title{
Somogy megye fullánkos hártyásszárnyú (Hymenoptera, Aculeata) faunája
}

\author{
JÓZAN ZSOLT
}

JóZAN Zs.: The Aculeata fauna of Somogy county (Hymenoptera, Aculeata)

Abstract: The checklist of the Aculeata fauna of Somogy county based on eight decade's researchwork is published by the author. In this area 992 species are known belonging to Scolioidea 29 , Chrysidoidea 75, Pompiloidea 77, Vespoidea 64, Sphecoidea 221 Apoidea 526. 72 percentage of the Hungarian Aculeata fauna occurs in Somogy county. Crossocerus walkeri is a new species in the Hungarian Aculeata fauna.

\section{A faunisztikai kutatások áttekintése}

Somogy megyében a hártyásszárnyú fauna vizsgálata a Balaton partja mentén kezdódött el. A 20. század első felében Méhely L. Balatonszemesen gyújtött, fóképpen méhszerú fajokat. Az elsố gyúitoőprogramot a Magyar Természettudományi Múzeum munkatársai, Bajári E., Móczár L., Móczár M., Solymosné a Kisbalaton területén folytatták. Ezt követóen a gyújtések Zamárdi környékére súlypontozódtak. A megye más területein, a publikált szórványos faunisztikai adatok tanúsága szerint, rendszeres faunisztikai feltáró munka nem történt. A szerzó a 60-as években kezdte a megye fullánkos faunájának vizsgálatát. Ez a munka az egymást követó kutatóprogramokhoz kapcsolódva teljesedett ki. Elsőként a Janus Pannonius Múzeum Természettudományi Osztálya által szervezett és irányított „A Mecsek környéke természeti képe" program keretében, majd később a Somogy Megyei Múzeum kutatásaihoz kapcsolódva. Ezek során részletes faunisztikai vizsgálatok folytak a Zselic, a Barcsi borókás TK, a Boronka-melléki TK, a Látrányi Puszta TT területén és a Baláta környékén. A 80-as években a szerző többször is részesült a Magyar Természettudományi Múzeum jóvoltából a Magyar Tudományos Akadémia kutató pedagógusok számára létesített ösztöndíjában, mely segítette Külső-Somogy, elsősorban a Balaton környéke fullánkos faunájának kutatását.

A régebbi gyújtések faunisztikai adatainak csak egy részét közölték a Hymenoptera faunakatalógusokban (Cat. Hym.), és a Magyarország Állatvilága (Fauna Hungariae) XIII. kötetének egyes füzeteiben (ld. irodalom). A szerző által folytatott kutatások eredményeinek nagy részét publikációkban dolgozta fel (JózAN 1971, 1983, 1985a, 1985b, 1990, 1992a, 1992b, 1995, 1996, 1998, 2000.). 


\section{A faunisztikai eredmények értékelése}

A fajjegyzék nem tartalmazza a Formicidae, Embolemidae, Bethylidae és a Dryinidae család fajait, mert ezek kutatására még nem került sor, irodalmi adatok sem állnak rendelkezésre. Nem vettük figyelembe a Pompilidae fajokra vonatkozó régebbi faunisztikai adatokat a szükségessé vált jelentős mértékú taxonómiai revízió miatt. A Zselicet egységes faunakistájként vettük figyelembe, néhány faj tulajdonképpen nem Somogy megye közigazgatási területén került elő.

A régebbi és az újabb gyứjtések során elókerült, illetve publikált fajok számát az l. táblázatban foglaljuk össze.

1 . táblázat: Somogyból elókerült Aculeata fajok száma családsorozatonként $A=$ elókerült fajok összesen, $B=$ a Magyarország Állatvilága füzeteiben és a Hymenoptera faunakatalógusokban közölt fajok száma, $\mathrm{C}=$ a még eddig nem publikált fajok száma

\begin{tabular}{|l|c|c|c|}
\hline Családsorozat & A & B & C \\
\hline Scolioidea (s. lato) & 29 & 15 & 6 \\
\hline Chrysidoidea & 75 & 4 & 16 \\
\hline Pompiloidea & 77 & 20 & 4 \\
\hline Vespoidea & 64 & 8 & 7 \\
\hline Sphecoidea & 221 & 54 & 40 \\
\hline Apoidea & 526 & 194 & 5 \\
\hline Összesen: & 992 & 295 & 48 \\
\hline
\end{tabular}

Az elókerült fajok száma jelentôs, a Magyarország területén eddig ismert fajok $72 \%$-át teszi ki.A jegyzékben szereplố fajok között számos olyan található, amelyik hazánk területéről a szerző gyújtéseinek során vált ismertté. Közel ötven olyan faj is található a jegyzékben, melynek Somogy megyei elófordulását eddig még sehol nem közölték. A Crossocerus walkeri (Sphecidae) hazánk faunájára is új. Somogysárdon került elő egy hím példánya, egy nóstényt pedig Újszentmargitán gyújtöttek. A fajjegyzékben a család értékú taxonok a hazai szakirodalomban alkalmazott családsorozatoknak felelnek meg, ezek szerepelnek a 2. táblázatban is.

2. táblázat: A Somogyból ismert taxonok és irodalmi hivatkozásuk $A$ = A faj lelóhelyeinek száma a megye területén, $B=A$ fajra vonatkozó publikációk sorszáma az irodalomjegyzékben

Fajok

Tiphiidae

Tiphia femorata Fabricius, 1775

Tiphia minuta Linden, 1827

Tiphia morio Fabricius, 1787

Tiphia ruficomis Klug, 1810

Meria tripunctata (Rossi, 1790)

Methoca ichneumonides Latreille, 1809
A
B

$1,26,30,31,32,33,34$ $31,32,34$ 30

$1,26,30,31,32,33,34$

$1,26,30,32,33$ 


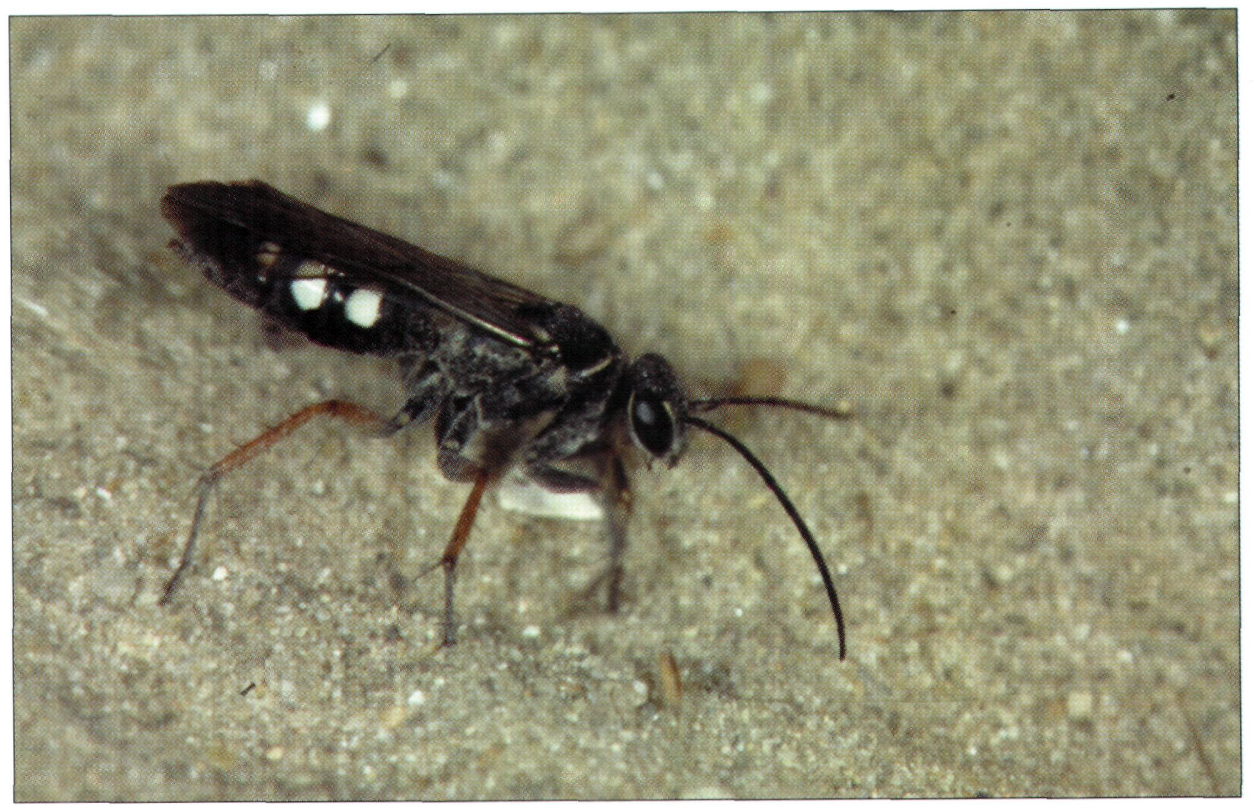

1. ábra: Piroslábú útonálló (Episyron rufipes) - Fotó: Móczár L.

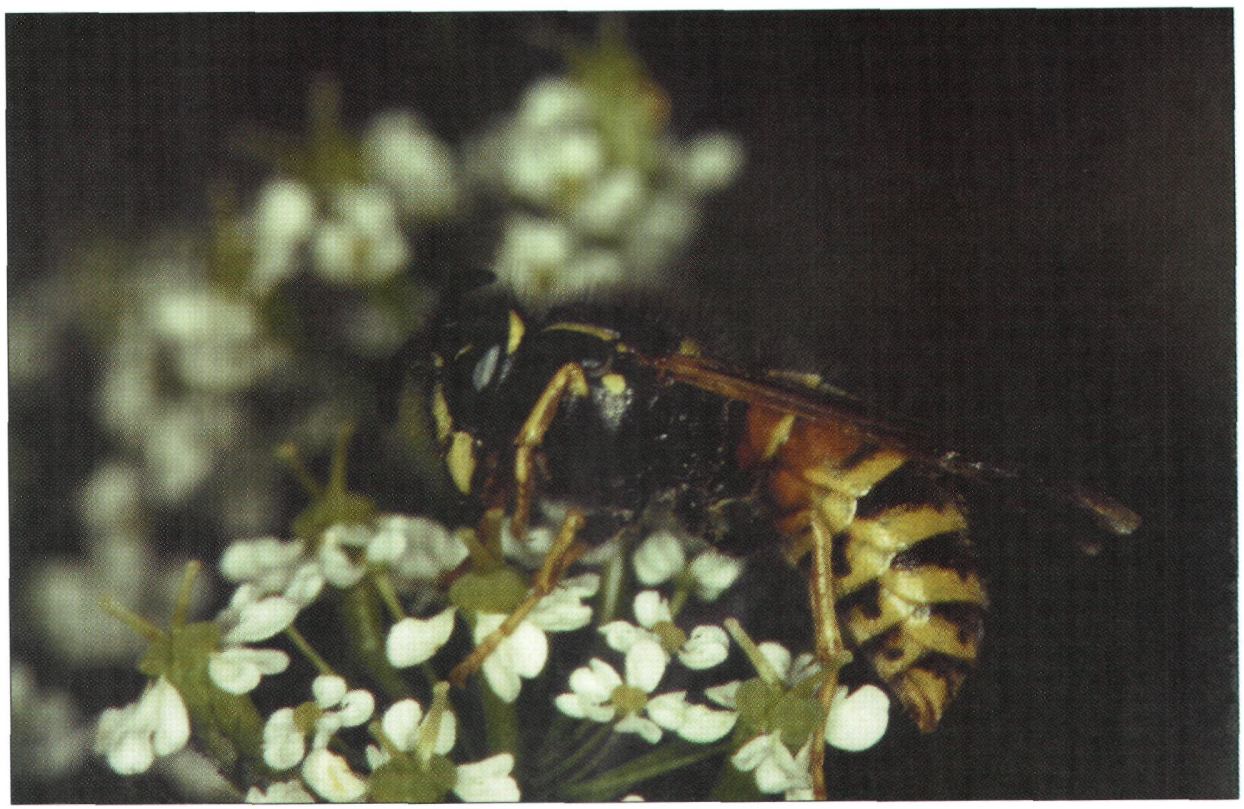

2. ábra: Pirosfoltos darázs (Vespula rufa) - Fotó: Móczár L. 


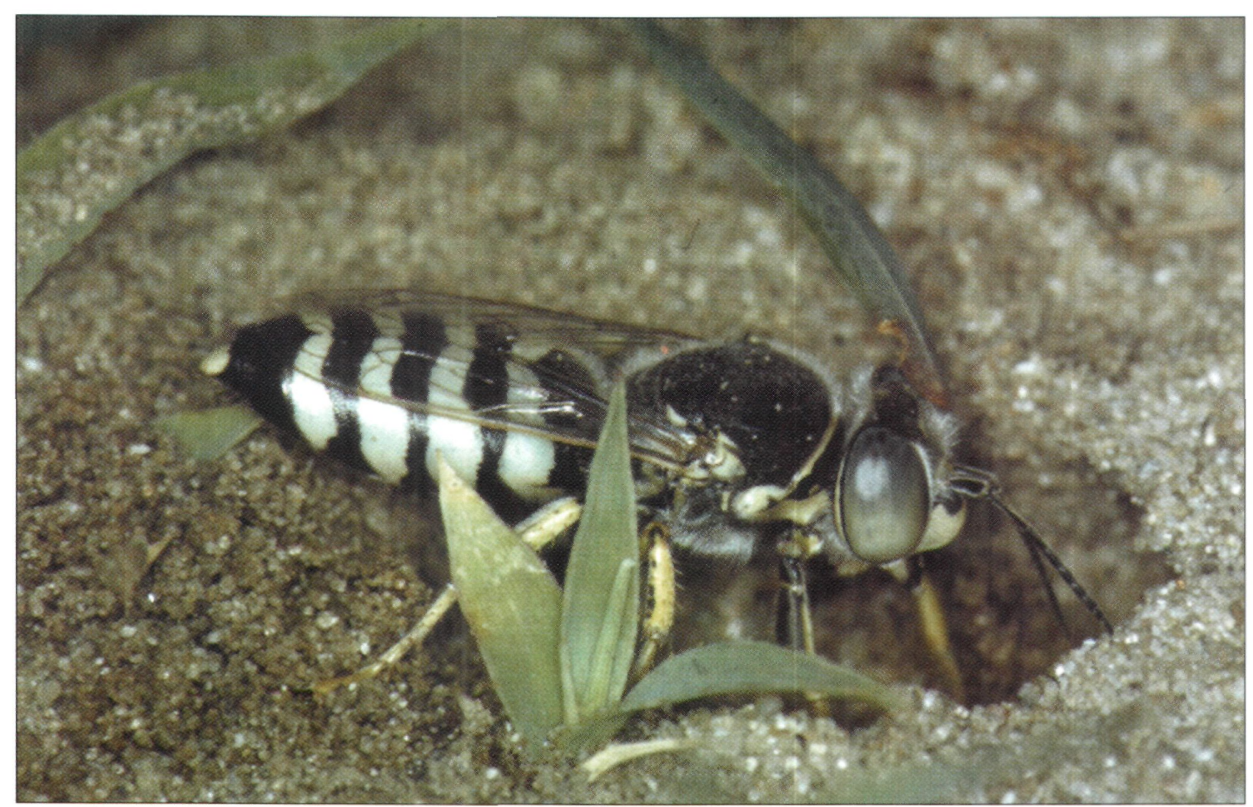

3. ábra: Óriás csôrôsdarázs (Bembix rostrata) Fotó: Móczár L.

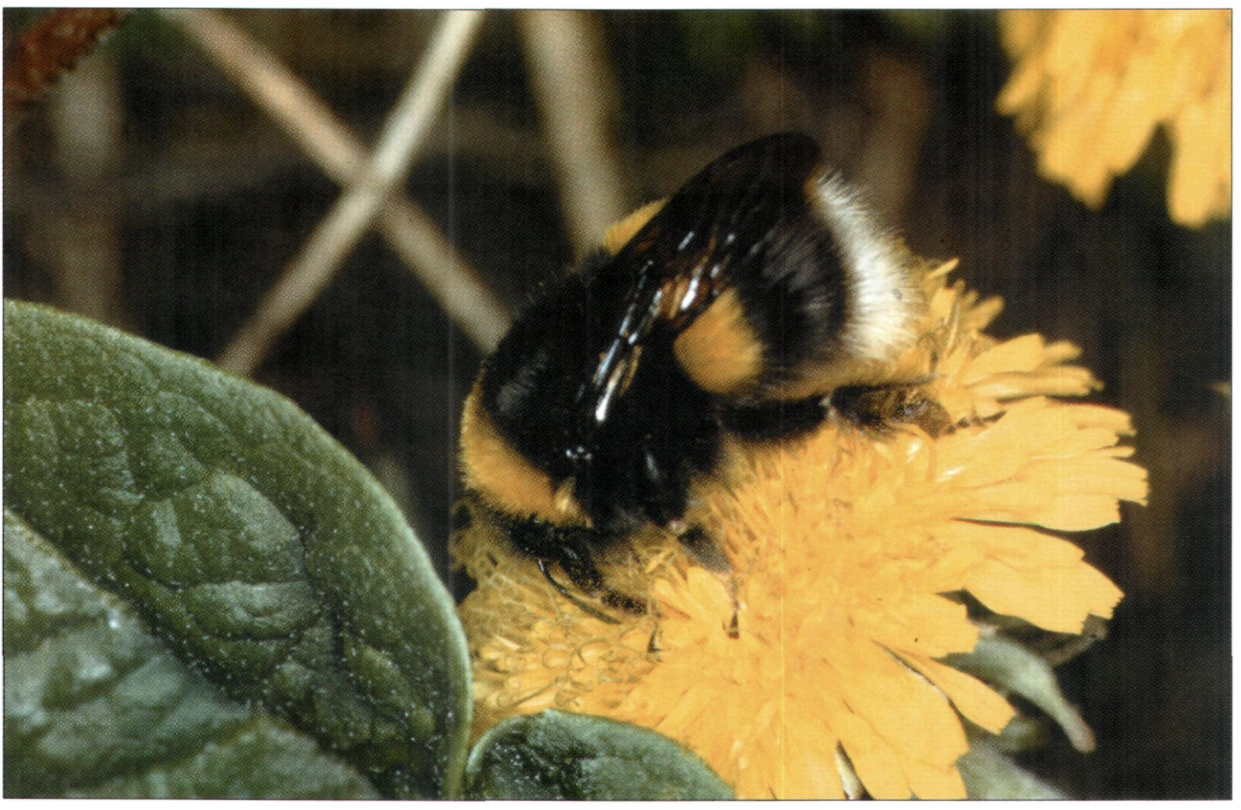

4. ábra: Földi poszméh (Bombus terrestris) Fotó: Móczár L. 


\section{Sapygidae}

Polochrum repandum Spinola, 1809

Sapyga clavicornis (Linné, 1758)

Sapyga quinquepunctata (Fabricius, 1781)

Sapygina decemguttata (Jurine, 1807)

\section{Scoliidae}

Scolia hirta (Schrank, 1781)

Scolia quadripunctata (Fabricius, 1775)

Campsoscolia interrupta (Fabricius, 1781)

\section{Myrmosidae}

Myrmosa brunnipes Lepeletier, 1845

Myrmosa melanocephala (Fabricius, 1793)

\section{Mutillidae}

Myrmilla calva (Villers, 1789)

Myrmilla mutica (André, 1893)

Mutilla europaea Linné, 1758

Smicromyrme cingulata (Costa, 1858(60))

Smicromyrme halensis (Fabricius, 1787)

Smicromyrme pusilla (Klug, 1835)

Smicromyrme punctata (Latreille, 1792)

Smicromyrme rufipes (Fabricius, 1787)

Smicromyrme scutellaris (Latreille, 1792)

Smicromyrme sicana (Destefani, I887)

Smicromyrme subcomata (Wesmael, 1852)

Smicromyrme piduata (Pallas, 1773)

Dasylabris maura (Linné, 1758)

Dasylabris regalis (Fabricius, 1793)

\section{Chysididae}

\section{Cleptinae}

Cleptes consimilis Buysson, 1887

Cleptes nitidulus (Fabricius, 1793)

Cleptes pallipes Lepeletier, I 806

Cleptes semiauratus (Linné, 1761)

\section{Chrysidinae}

Elampus bidens (Förster, 1853)

Elampus constrictus (Förster, 1853)

Elampus scutellaris (Panzer, 1798)

Omalus aeneus (Fabricius, 1787)

Omalus biaccinctus (Buysson, 1893)

Philoctetes truncatus (Dahlbom, 1831)

Pseudomalus auratus (Linné, 1758)

Pseudomalus bidentulus (Lepeletier, 1806)

Pseudomalus bogdanovi (Radoszkowski, 1877)

Pseudomalus pusillus (Fabricius, 1804)

Pseudomalus violaceus (Scopoli, 1763)

Holopyga amoenula Dahlbom, 1834

Holopyga chrysonota (Förster, 1853)

Holopyga fervida (Fabricius, 1781)

Holopyga minuma Linsenmaier, 1959)

Hedychrum aureicolle Mocsáry, 1889

Hedychrum gerstaeckeri Chevrier, 1869
2

$4 \quad 34$

$41 \quad 1,30,31,32,33,34$

$23 \quad 1,30,31,32,34$

17

31,30

42

34

$1,26,30,31,32,33,34$

$1,26,30,31,32,33,34$

25

4

2

3

6

1

3

39

1

I

2

5

7

5

$12,31,32$

31

12

$12,26,30,31,32$

26

$26,30,31,32,33,34$

32

$12,26,30,32$

$26,30,33,34$

12,30

34

30,32

$30,33,34$

31,34

30,33

$31,32,34$

31

$30,31,32,33,34$

$30,31,34$

33,34

$30,31,32,33,34$

$31,32,33$

$10: 31,32$

4

3

42

30,34

$30,31,32,34$

$50 \quad 30,31,32,33,34$ 
Hedychrum nobile (Scopoli, 1763)

66

Hedychrum rutilans (Dahlbom, 1854)

Hedychridium ardens (Coquebert, 1801)

Hedychridium aheneum (Dahlbom, 1854)

Hedychridium coriaceum (Dahlbom, 1854)

Hedychridium elegantulum Buysson, 1887

Hedychridium femoratum (Dahlbom, 1854)

Hedychridium jucundum (Mocsáry, 1880)

Hedychridium krajniki Balthasar, 1946

Hedychridium monochroum Buysson, 1888

Hedychridium roseum (Rossi, 1790)

Hedychridium scutellare Tournier, 1878

Hedychridium zelleri (Dahlbom, 1845)

Parnopes grandior (Pallas, 1771)

Brugmoia quadrata (Shuckard, 1836)

Spinolia unicolor (Dahlbom, 1831)

Spinolia dallatorreana (Mocsáry, I896)

Pseudospinolia neglecta (Shuckard, 1837)

Chrysidea pumila (Klug, 1845)

Chrysura cuprea (Rossi, I790)

Chrysura dichroa (Dahlbom, 1854)

Chrysura radians (Harris, 1776)

Chrysura rufiventris (Dahlbom, 1854)

Chrysura trimaculata (Förster, 1853)

Chrysis angustifrons Abeille, 1878

Chrysis angustula Schenck, 1856

Chrysis cingulicornis Förster, 1853

Chrysis compta Förster, 1853

Chrysis distincta Olivier, 1790

Chrysis fasciata Olivier, 1790

Chrysis fulgida Linné, 1761

Chrysis germari Wesmael, 1839

Chrysis gracillima Förster, 1853

Chrysis graelsii Guerin, 1842

Chrysis grohmanni Dahlbom, 1854

Chrysis ignita (Linné, 1758)

Chrysis inaequalis Dahlbom, 1854

Chrysis indigotea (Dufour et Perris, 1840)

Chrysis insperata Chevrier, 1870

Chrysis interjecta Buysson, 1895

Chrysis leachii Shuckard, 1836

Chrysis longula Abeille, 1878

Chrysis marginata Mocsáry, 1889

Chrysis pseudobrevitarsis Linsenmaier, 1951

Chrysis pulchella Spinola, 1808

Chrysis ragusae Destefani, 1888

Chrysis rambouri Dahlbom, 1854

Chrysis rutilans Olivier, 1790

Chrysis rutiliventris Abeille, 1879

Chrysis scutellaris Fabricius, 1794

Chrysis subsinuata Marquet, 1879

Chrysis succincta Linné, 1767

Chrysis viridula Linné, 1761

Trichrysis cyanea (Linné, 1758)
$30,31,32,33,34$

$30,31,32,33,34$

$30,31,32,34$

$30,31,32,33,34$

5,34

$30,31,32,33,34$

$30,31,32,33,34$

33

30,33

$30,32,33,34$

30

31,33

$30,31,32,33,34$

3 I

$30,31,32,33,34$

$30,31,32,34$

31

32,34

31,34

34

$30,31,32,33,34$

$30,31,33$

$30,31,33,34$

$31,32,33,34$

$31,33,34$

$30,31,32,33,34$

$32,33,34$

$30,31,32,33$

32

$30,31,33,34$

$31,32,33$

33

30

31,32

$30,31,32,33,34$

33

$30,31,33,34$

$30,31,32,33,34$

34

$30,31,32,33,34$

$30,31,33$

$30,31,32,33,34$ 


\section{Pompilidae}

Pompilinae

Cryptocheilus fabricii (Linden, 1827)

Cryptocheilus notatus affinis (Linden, 1827)

Cryptocheilus versicolor versicolor (Scopoli, 1763)

Cryptocheilus versicolor vorticosus Haupt, 1927

Priocnemis agilis (Shuckard, 1837)

Priocnemis coriacea Dahlbom, 1843

Priocnemis enslini Haupt, 1927

Priocnemis exaltata (Fabricius, 1776)

Priocnemis fastigiata Haupt, 1934

Priocnemis fennica Haupt, 1927

Priocnemis gracilis Haupt, 1927

Priocnemis hankoi Móczár, 1944

Priocnemis hyalinata (Fabricius, 1793)

Priocnemis melanosoma Kohl, 1880

Priocnemis mimula Wesmael, 1851

Priocnemis minuta (Linden, 1827)

Priocnemis parvula Dahlbom, 1845

Priocnemis perturbator (Harris, 1776)

Priocnemis pillichi Priesner, 1960

Priocnemis pusilla Schiödte, 1837

Priocnemis schioedtei Haupt, 1927

Priocnemis sulci Balthasar, 1943

Priocnemis susterai Haupt, 1927

Caliadurgus fasciatellus (Spinola, 1808)

Dipogon bifasciatus (Geoffroy, 1785)

Dipogon monticolus Wahis, 1972

Dipogon subintermedius (Magretti, 185)

Dipogon variegatus (Linné, 1758)

Dipogon vechti Day, 1979

Poecilagenia sculpturata (Kohl, 1898)

Auplopus albifrons (Dalman, I823)

Auplopus carbonarius (Scopoli, 1763)

Auplopus rectus (Haupt, 1926)

Aporus femoralis Linden, 1827

Eoferreola manticata (Pallas, 1771)

Homonotus balcanicus Haupt, 1927

Pompilus cinereus (Fabricius, 1775)

Arachnospila abnormis (Dahlbom, 1842)

Arachnospila anceps (Wesmael, 1851)

Arachnospila ausa (Tournier, 1890)

Arachnospila fumipennis (Zetterstedt, 1838)

Arachnospila fuscomarginata (Thomson, 1870)

Arachnospila gibbomima (Haupt, 1929)

Arachnospila minutula (Dahlbom, 1843)

Arachnospila spissa (Schiödte, 1837)

Arachnospila trivialis (Dahlbom, 1843)

Arachnospila wesmaeli (Thomson, 1870)

Agenioideus cinctellus (Spinola, 1808)

Agenioideus sericeus (Linden, 1827)

Nanoclavelia leucoptera (Dahlbom, 1845)

Aporinellus moestus sericeomaculatus (Kohl, 1888)

Aporinellus obtusus (Gussakovskij, 1935)

Aporinellus sexmaculatus (Spinola, 1805)
30,34

$1430,31,32,33,34$

$20 \quad 31,32,33$

131

$7 \quad 34$

$1530,31,32$

$5 \quad 30,33$

$6 \quad 30,31,33$

130

134

$7 \quad 30,34$

$1630,31,32,33,34$

$10 \quad 30,32,34$

$8 \quad 30,31$

930,31

$630,31,33$

$59 \quad 30,31,32,33,34$

1

27

1
3 I

31,32

30,31

$30,31,34$

$30,31,34$

30,34

30

34

31

$30,31,32,33,34$

31,34

$30,31,32$

$30,32,33,34$

$30,31,34$

$30,31,32,33,34$

32

$3,30,32$

30

$30,31,32,33,34$

$30,31,32,33,34$

$30,31,32,34$

$30,32,33$

$30,31,32,33,34$

$30,31,32,33,34$

31,32

32

32

30,32 
Anospilus orbitalis (Costa, 1863)

Evagetes crassicornis (Shuckard, 1845)

Evagetes dubius (Linden, 1827)

Evagetes gibbulus (Lepeletier, 1845)

Evagetes littoralis (Wesmael, 1851)

Evagetes elongatus (Lepeletier, 1845)

Evagetes pectinipes (Linné, 1758)

Eragetes siculus (Lepeletier, 1845)

Evagetes tumidosus (Tournier, 1890)

Anoplius alpinobalticus Wolf, 1956

Anoplius caviventris (Aurivillius, 1907)

Anoplius concinnus (Dahlbom, I845)

Anoplius infuscatus (Linden, 1827)

Anoplius nigerrimus (Scopoli, 1763)

Anoplius piliventris (Morawitz, 1889)

Anoplius viaticus paganus (Dahlbom, 1843)

Episyron albonotatus (Linden, 1827)

Episyron arrogans (Smith, 1873)

Episyron rufipes (Linné, 1758)

Batazonellus lacerticida (Pallas, 1771)

Telostegus inermis (Brullé, 1832)

\section{Ceropalinae}

Ceropales helvetica Tournier, 1889

Ceropales maculata (Fabricius, 1775)

Ceropales variegata (Fabricius, I798)

\section{Vespidae}

Vespinae

Vespa crabro Linné, 1758

Vespula germanica (Fabricius, 1793)

Vespula rufa (Linné, 1758)

Vespula vulgaris (Linné, 1758)

Dolichovespula media (Retzius, I783)

Dolichorespula saxonica (Fabricius, 1793)

Dolichovespula sylvestris (Scopoli, 1763)

Polistes associus Kohl, 1898

Polistes bischoffi (Weyrauch, 1939)

Polistes dominulus (Christ, 1791)

Polistes nympha (Christ, 1791)

Polistes omissus (Weyratuch, 1939)

\section{Eumeninae}

Discoelius dufourii Lepeletier, 1841

Discoelius zonalis (Panzer, 1801)

Odynerus femoratus Saussure, 1856

Odynerus melanocephalus (Gmelin, 1790)

Odynerus poecilus Saussure, 1856

Odynerus reniformis (Gmelin, 1790)

Odynerus spinipes (Linné, 1758)

Gymnomerus laevipes (Shuckard, 1837)

Pterocheilus phaleratus (Panzer, I797)

Alastor biegelebeni Giordani Soika, 1942

Pseudomicrodynerus parrulus (Herrich-Schaeffer, I838)

Microdynerus nugdunensis (Saussure, 1856)

Microdynerus timidus (Saussure, 1856)
$1 \quad 32$

$3 \quad 31,32,34$

$8 \quad 30,32,33,34$

630,33

$930,31,32$

$4 \quad 31$

$10 \quad 30,32,33,34$

$5 \quad 31,32$

$1 \quad 3$

23,32

$6 \quad 3,34$

$5 \quad 30,3 \mathrm{I}, 33$

$21 \quad 30,31,32,33$

$31 \quad 30,31,32,33,34$

13

$32 \quad 30,31,32,33,34$

$13 \quad 30,32,34$

$20 \quad 30,31,32,34$

$24 \quad 30,31,32,33,34$

1

$3 \quad 30,33$

23,30

$21 \quad 30,31$

1

27

$30,31,32,33,34$

$36 \quad 30,31,32,33,34$

$4 \quad 31,32$

$17 \quad 30,31,32,33,34$

$10 \quad 32,33$

$5 \quad 31$

$5 \quad 33$

26

$10 \quad 32$

$32 \quad 30,31,32,33,34$

$38 \quad 30,31,32,33,34$

1
30,31

6

31,32

31

$30,31,32,33$

$30,31,33,34$

$30,31,32,33,34$

$30,32,33,34$

$30,31,33,34$

$30,31,32,34$

$30,31,32,33,34$

$30,31,33,34$ 
Leptochilus alpestris (Saussure, 1856)

5

Leptochilus regulus (Saussure, 1856)

Stenodynerus bluethgeni v. d. Vecht, 1971

Stenodynerus chevrieranus (Saussure, 1856)

Stenodynerus clypeopictus (Kostylev, 1940)

Stenodynerus orenburgensis (André, 1884)

Stenodynerus steckianus (Schulthess, 1897)

Stenodynerus xanthomelas (Herrich-Schaeffer, 1839)

Allodynerus delphinalis (Giraud, 1866)

Allodynerus rossii (Lepeletier, 1841)

Antepipona orbitalis (Herrich-Schaeffer, 1839)

Parodontodynerus ephippium (Klug, 1817)

Euodynerus dantici (Rossi, 1790)

Euodynerus egregius unimaculatus (Maidl, 1922)

Euodynerus notatus (Jurine, 1807)

Euodynerus posticus (Herrich-Schaeffer, 1841)

Euodynerus quadrifasciatus (Fabricius, I793)

Jucancistrocerus jucundus (Mocsáry, 1883)

Ancistrocerus acutus (Fabricius, 1793)

Ancistrocerus claripennis Thomson, 1874

Ancistrocerus gazella (Panzer, 1798)

Ancistrocerus ichneumonideus (Ratzeburg, 1844)

Ancistrocerus nigricornis (Curtis, 1826)

Ancistrocerus parietinus (Linné, 1761)

Ancistrocerus parietum (Linné, 1758)

Ancistrocerus trifasciatus (Müller, 1776)

Symmorphus bifasciatus (Linné, 1761)

Symmorphus connexus (Curtis, 1826)

Symmorphus crassicornis (Panzer, 1798)

Symmorphus debilitatus (Saussure, 1855)

Symmorphus gracilis (Brullé, 1832)

Symmorphus murarius (Linné, 1758)

Eumenes coronatus (Panzer, 1799)

Eumenes coarctatus (Linné, 1758)

Eumenes dubius Saussure, 1852

Eumenes lunulatus Fabricius, 1804

Eumenes mediterraneus Kriechbaumer, 1879

Eumenes papillarius (Christ, 1791)

Eumenes pedunculatus (Panzer, 1799)

\section{Sphecidae}

\section{Sphecinae}

Ampulex fasciata Jurine, 1807

Dolichurus corniculus (Spinola, 1808)

Chalybion femoratum (Fabricius, 1781)

Sceliphron curvatum (Smith, 1870)

Sceliphron destillatorium (Illiger, 1807)

Sceliphron spirifex (Linné, 1758)

Sphex rufocinctus Brullé, 1833

Prionyx kirbyi (Linden, 1827)

Ammophila campestris Latreille, 1809

Ammophila heydeni Dahlbom, 1845

Ammophila sabulosa (Linné, 1758)

Ammophila terminata mocsaryi Frivaldszky, 1976

Podalonia affinis (Kirby, 1798)
$30,31,32,33,34$

$30,31,34$

$30,31,32,34$

6,32

6

32

$30,31,32,34$

$30,32,33,34$

31,32

$30,31,33,34$

$30,33,34$

31

6

$30,31,32,33,34$

30,31

$30,32,33$

31,34

$31,32,33,34$

$31,32,33,34$

30,34

$30,31,32,33,34$

34

$30,32,34$

$30,31,32,34$

34

$30,31,32,34$

34

$30,31,32,34$

$30,31,32,34$

32,33

30,34

30,34

$30,31,32,33,34$

33

$26,28,31,32$

$30,31,33$

34

$26,31,32,33,34$

2,28

$28,30,32,33,34$

$28,30,32,33,34$

$26,27,28,30,31,32,33,34$

$26,28,31,32$

$26,27,30,31,32,33,34$

$26,27,28,30,32,33,34$

28 
Podalonia luffi (Saunders, 1903)

Podalonia hirsuta (Scopoli, 1763)

\section{Pemphredoninae}

Mimesa caucasia Maidl, 1914

Mimesa equestris (Fabricius, 1804)

Mimesa rufa (Panzer, 1805)

Mimumesa atratina (Morawitz, 1891)

Mimumesa dahlbomi (Wesmael, 1852)

Mimumesa littoralis (Bondroit, 1934)

Mimumesa unicolor (Linden, 1829)

Psen ater (Olivier, 1792)

Psenulus concolor (Dahlbom, 1843)

Psenulus fuscipennis (Dahlbom, 1843)

Psenulus laevigatus (Schenck, 1857)

Psenulus pallipes (Panzer, 1798)

Psenulus schencki (Tournier, 1889)

Diodontus insidiosus Spooner, 1938

Diodontus luperus Shuckard, 1837

Diodontus major Kohl, 1901

Diodontus medius Dahlbom, 1845

Diodontus minutus (Fabricius, 1793)

Diodontus tristis (Linden, 1829)

Pemphredon brevipetiolatus Wagner, 1931

Pemphredon clypealis Thomson, 1870

Pemphredon inornatus Say, 1824

Pemphredon lethifer (Shuckard, 1837)

Pemphredon lugubris (Fabricius, 1793)

Pemphredon lugens Dahlbom, 1842

Pemphredon morio Linden, 1829

Pemphredon rugifer Dahlbom, 1844

Passaloecus clypealis Faester, 1947

Passaloecus comiger Shuckard, 1837

Passaloecus gracilis (urtis, 1834

Passaloecus insignis (Linden, 1829)

Passaloecus singularis Dahlbom, 1844

Polemistus abnormis (Kohl, 1888)

Stigmus pendulus Panzer, 1804

Stigmus solskyi Morawitz, 1864

Spilomena mocsaryi Kohl, 1898

Spilomena troglodytes (Linden, 1829)

Ammoplanus handlirschi Gussakovskij, 1931

Ammoplanus hofferi Snoflak, 1943
$28,30,33$

31

$28,30,31$

$30,33,34$

$\begin{array}{ll}9 & 2,28,30,31,33 \\ 22 & 28,30,33,34\end{array}$

22

23

23

7

24

17

75

13

1

31

1

1

105

58

1

4

66

115

12

5

5

16

2

22

16

10

48

3

26

37

8

25

9

25

$27,28,30,31,32,34$

$26,31,32$

$28,31,32$

$26,28,30,31,32,33,34$

$28,30,34$

$26,28,30,31,32,33,34$

$30,31,32,34$

$28,30,31,32,33,34$

$26,28,30,31,32,33,34$

$28,30,31,32,33,34$

$28,30,32,34$

$28,30,31,32,33,34$

$26,27,28,30,31,32,33,34$

$28,30,31,34$

$28,30,34$

33,34

$28,30,31,32,33,34$

28

$28,30,33,34$

$26,28,30,34$

28,34

$28,30,31,32,33,34$

31,34

28,34

$28,30,32,33,34$

30,34

$28,30,31,34$

$30,31,33$

31,34

\section{Astatinae}

Astata apostata Mercet, 1910

Astata boops (Schrank, 1781)

Astata costae Piccioli, 1867

Astata gallica Beaumont, 1942

Astata kashmirensis Nurse, 1909

Astata minor Kohl, 1885

Dryudella tricolor (Linden, 1829)

Dinetus pictus (Fabricius, 1793)
1

\section{2,28}

$26,28,30,31,32,33$

2,30

$28,31,32$

$26,28,30,31,32,33,34$

30

$26,28,30,31,32,33,34$ 


\section{Larrinae}

Larra anathema (Rossi, 1790)

Liris nigra (Fabricius, 1775)

Tachytes etruscus (Rossi, 1790)

Tachytes europaeus Kohl, 1884

Tachytes obsoletus (Rossi, 1792)

Tachysphex bicolor Brullé, 1856

Tachysphex fugax (Radoszkowski, 1877)

Tachysphex fulvitarsis (Costa, 1867)

Tachysphex grandii Beaumont, 1965

Tachysphex helveticus Kohl, 1885

Tachysphex mocsaryi Kohl, 1884

Tachysphex nitidus (Spinola, 1805)

Tachysphex obscuripennis (Schenck, 1857)

Tachysphex panzeri (Linden, 1829)

Tachysphex pompiliformis (Spinola, 1804)

Tachysphex psammobius (Kohl, 1880)

Tachysphex tarsinus (Lepeletier, 1845)

Palarus variegatus (Fabricius, 1781)

Solierella compedita (Piccioli, 1869)

Miscophus ater Lepeletier, 1845

Miscophus bicolor Jurine, 1807

Miscophus concolor Dahlbom, 1844

Miscophus spurius (Dahlbom, 1832)

Nitela fallax Kohl, 1884

Nitela spinolae Latreille, 1809

Pison atrum (Spinola, 1808)

Trypoxylon attenuatum Smith, 1851

Trypoxylon clavicerum Lepeletier et Serville, 1828

Trypoxylon figulus (Linné, 1758)

Trypoxylon fronticome Gussakovskij, 1936

Trypoxylon scutatum Chevrier, 1867

\section{Crabroninae}

Belomicrus antennalis Kohl, 1899

Oxybelus argentatus Curtis, 1833

Oxybelus aurantiacus Mocsáry, 1883

Oxybelus bipunctatus Olivier, 1811

Oxybelus latidens Gerstaecker, 1867

Oxybelus latro Olivier, 1811

Oxybelus lineatus (Fabricius, 1787)

Oxybelus mandibularis Dahlbom, 1845

Oxybelus mucronatus (Fabricius, 1793)

Oxybelus quattordecimnotatus Jurine, 1807

Oxybelus trispinosus (Fabricius, 1787)

Oxybelus uniglumis (Linné, 1758)

Oxybelus variegatus Wesmael, 1853

Oxybelus victor Lepeletier, 1845

Entomognathus brevis (Linden, 1829)

Entomognathus dentifer (Noskiewicz, 1929)

Lindenius albilabris (Fabricius, 1793)

Lindenius laevis Costa, 1871

Lindenius panzeri (Linden, 1829)

Lindenius parkanensis Zavadil, 1948

Lindenius pygmaeus armatus (Linden, 1829)
10

3

11

27

2

3

1

8

4

20

2

51

11

\section{2}

$28,30,33,34$

31,34

$26,28,30,34$

$26,28,30,31,32,33,34$

26,28

$26,27,28,30$

31,34

$28,31,33,34$

$30,31,32,33$

$28,30,32,33$

$28,30,32$

$26,27,28,30,31,32,33,34$

$26,27,28,30,32,33,34$

$26,28,30,32$

$26,27,28,30,31,32,33,34$

$27,28,30,31,32,33,34$

30,31

$26,27,28,30,31,32,33,34$

32,33

33

$26,27,28,30,31,32,33,34$

31

$26,27,28,30,31,32,33,34$

$30,31,34$

$28,30,32,33,34$

$28,30,31,32,33,34$

$28,30,31,32,34$

$28,30,31,32,33,34$

$26,28,30,31,32,33,34$

30

$26,28,31,32,33,34$

30

$26,27,28,30,32,33$

$4,17,26,28,32$

$17,26,28,30,31,32,33,34$

17,28

$4,17,28$

$4,17,26,28,32$

$17,26,28,30,32,33$

$17,28,30,31$

$17,26,27,28,30,31,32,33,34$

$26,28,30,31,33$

$26,28,30,31,32,33,34$

$17,26,28,30,31,33,34$

$17,28,30,31,34$

I $7,28,29,30,31,32,33,34$

28

$17,26,27,28,30,31,32,33,34$

$28,31,32,34$

$26,28,30,31,32$

$4,17,28$

$17,28,30,31,32,34$ 
Rhopalum austriacum (Kohl, 1899)

Rhopalum clavipes (Linné, 1758)

Rhopalum coarctatum (Scopoli, 1763)

Rhopalum gracile Wesmael, 1852

Crossocerus acanthophorus (Kohl, 1892)

Crossocerus annulipes (Lepeletier et Brullé, 1834)

Crossocerus assimilis (Smith, 1856)

Crossocerus binotatus Lepeletier et Brullé, 1834

Crossocerus capitosus (Shuckard, 1837)

Crossocerus cetratus (Shuckard, 1837)

Crossocerus distinguendus (Morawitz, I866)

Crossocerus elongatulus (Linden, 1829)

Crossocerus exiguus (Linden, 1829)

Crossocerus megacephalus (Rossi, 1790)

Crossocerus nigritus (Lepeletier et Brullé, 1834)

Crossocerus ovalis Lepeletier et Brullé, 1834

Crossocerus palmipes (Linné, 1767)

Crossocerus podagricus (Linden, 1829)

Crossocerus quadrimaculatus (Fabricius, 1793)

Crossocerus tarsatus (Shuckard, 1837)

Crossocerus vagabundus (Panzer, 1798)

Crossocerus varius Lepeletier et Brullé, 1834

Crossocerus walkeri (Shuckard, 1837)

Crossocerus wesmaeli (Linden, 1829)

Tracheliodes curritarsis (Herrich-Schaeffer, 1841)

Crabro cribrarius (Linné, 1758)

Crabro peltarius (Screber, I784)

Crabro scutellatus (Scheven, 1781)

Ectemnius cavifrons (Thomson, 1870)

Ectemnius cephalotes (Olivier, 1792)

Ectemnius confinis (Walker, 1871)

Ectemnius continuus (Fabricius, 1804)

Ectemnius dives (Lepeletier et Brullé, 1834)

Ectemnius fossorius (Linné, 1758)

Ectemnius guttatus (Linden, 1829)

Ectemnius lapidarius (Panzer, 1804)

Ectemnius lituratus (Panzer, 1804)

Ectemnius meridionalis (Costa, 187I)

Ectemnius nigritarsus (Herrich-Schaeffer, I84I)

Ectemnius rubicola (Dufour et Perris, 1840)

Ectemnius ruficornis (Zetterstedt, 1838)

Ectemnius rugifer (Dahlbom, 1845)

Ectemnius schlettereri (Kohl, 1888)

Lestica alata (Panzer, 1797)

Lestica clypeata (Schreber, 1759)

\section{Nyssoninae}

Mellinus arvensis (Linné, 1758)

Alysson spinosus (Panzer, 1801)

Alysson tricolor Lepeletier et Serville, 1825

Didineis crassicornis Handlirsch, 1888

Didineis lunicornis (Fabricius, 1798)

Nysson dimidiatus Jurine, 1807

Nysson fulpipes Costa, 1859

Nysson interruptus (Fabricius, 1798)
34

34

$28,30,32,34$

$4,17,28,30,34$

$30,31,34$

$28,30,32,34$

$28,30,34$

28

28,32

$26,28,30,3 \mathrm{I}, 34$

$28,30,31,33,34$

$17,28,30,31,33,34$

$26,28,30,31,33,34$

$17,28,30,34$

$28,31,34$

$4,17,28,30,31$

$4,17,28$

$26,28,30,31,32,33,34$

$17,28,30,31,32,33,34$

31

$26,28,33,34$

30

$4,17,26,28,30,32,33,34$

$17,26,28,30,31,32,33,34$

$17,26,27,28,30,32,34$

$4,17,26,28,30,32,33,34$

$26,28,30,31,32,33,34$

$17,28,31,34$

$17,28,30,34$

$17,26,27,28,30,31,32,33,34$

$17,26,28,30,31,32,33,34$

$17,26,28,30,31,32,34$

$26,28,30,31,33,34$

$17,26,28,30,31,32,33,34$

$28,30,31,33,34$

$17,28,30,31,34$

32

$17,26,28,30,31,32,33,34$

$26,28,30,31,33$

$26,28,30,31,32,33,34$

$4,17,28$

$17,26,28,30,31,32,33$

$17,26,28,30,31,32,33,34$

16

57

2

1

5

24

1

12
$26,28,30,32,34$

$26,28,30,3 \mathrm{I}, 32,33,34$

28,31

$26,27,28,30,31,32,33$

2

28,31 
Nysson maculosus (Gmelin, 1790)

Nysson niger Chevrier, 1868

Nysson spinosus (Forster, 1771)

Nysson tridens Gerstaecker, 1867

Nysson trimaculatus (Rossi, 1790)

Brachystegus scalaris (Illiger, 1807)

Argogorytes fargeii (Shuckard, 1837)

Argogorytes mystaceus (Linné, 1761)

Dienoplus affinis (Spinola, 1808)

Dienoplus elegans (Lepeletier, 1832)

Dienoplus exiguus (Handlirsch, 1888)

Dienoplus laeris (Latreille, 1792)

Dienoplus lunatus (Dahlbom, 1832)

Dienoplus moravicus (Snoflak, 1946)

Dienoplus tumidus (Panzer, 1801)

Gorytes fallax Handlirsch, 1888

Gorytes laticinctus (Lepeletier, 1832)

Gorytes pleuripunctatus (Costa, 1859)

Gorytes procrustes Handlirsch, 1888

Gorytes quadrifasciatus (Fabricius, 1804)

Gorytes quinquecinctus (Fabricius, 1793)

Gorytes quinquefasciatus (Panzer, 1798)

Gorytes sulcifrons (Costa, I869)

Lestiphorus bicinctus (Rossi, 1794)

Lestiphorus bilunulatus Costa, 1869

Hoplisoides craverii (Costa, 1869)

Hoplisoides latifrons (Spinola, 1808)

Hoplisoides punctuosus (Eversmann, 1849)

Bembecinus hungaricus (Frivaldszky, 1876)

Bembecinus tridens (Fabricius, 1781)

Bembix megerlei Dahlbom, 1845

Bembix oculata Panzer, 1801

Bembix rostrata (Linné, 1758)

Bembix tarsata Latreille, 1809

\section{Philanthinae}

Philanthus coronatus (Thunberg, 1784)

Philanthus triangulum (Fabricius, 1775)

Philanthus venustus (Rossi, 1790)

Cerceris albofasciata (Rossi, 1790)

Cerceris arenaria (Linné, 1758)

Cerceris bicincta Klug, I 835

Cerceris bracteata Eversmann, 1849

Cerceris circularis dacica Schletterer, 1887

Cerceris flavilabris (Fabricius, 1793)

Cerceris hortivaga Kohl, 1880

Cerceris interrupta (Panzer, 1799)

Cerceris lunata funerea Costa, 1869

Cerceris quadricincta (Panzer, 1799)

Cerceris quadrifasciata (Panzer, 1799)

Cerceris quinquefasciata (Rossi, I792)

Cerceris rubida (Jurine, 1807)

Cerceris ruficomis (Fabricius, 1793)

Cerceris rybyensis (Linné, 1771)

Cerceris sabulosa (Panzer, 1799)

Cerceris somotorensis Balthasar, 1956
18

11

23

4

20 .

4

3

27

26

14

1

17

1

2

3

22

34

7

3

35

82

2

2

\section{1}

\section{6}

\section{1}

3

5

12

32

1

2

23

2

5

63

2

24

92

3

1

4

9

30

3

1

9

8

65

5

25

30

85

1
$28,30,31,32,33$

$26,28,30,32,33$

$28,30,31,32,34$

28,32

$2,26,28,30,31,32,34$

$28,30,31$

28

$28,30,31,32$

$27,28,30,31,34$

$26,28,30,31,32,33,34$

32

$28,32,33$

33

28,33

$28,30,31,32,33,34$

$26,28,30,31,32,34$

28,31

2,28

$28,30,31,32,33,34$

$26,28,30,31,32,33$

30

2

2,28

$28,31,32$

2,28

28,31

$30,31,34$

$26,27,28,30,32,33,34$

$26,27,28,30,31,32,33,34$ 28

$26,28,30,32$

$26,28,30,31,32,33,34$

28

\section{8,31}

$26,28,30,31,32,33,34$

30

$2,14,26,28,30,31,32,34$

$26,27,28,30,31,32,33,34$

I4, 28

14

$14,26,28,30,34$

I4, 28, 31, 33

$26,27,28,30,31,32,34$

$14,28,31$

14,28

$2,14,31,34$

28,32

$26,28,30,31,32,33,34$

$2,14,28$

$26,28,31,32,34$

$26,27,28,30,31,32,33,34$

$26,27,28,30,31,32,33,34$ 28 


\section{Apidae}

Colletinae

Hylaeus angustatus (Schenck, 1859)

Hylaeus annularis (Kirby, 1802)

28

Hylaeus brevicornis Nylander, 1852)

Hylaeus cardioscapus Cockerell, 1924

Hylaeus communis Nylander, 1852

Hylaeus cornutus Curtis, 1831

Hylaeus difformis (Eversmann, 1852)

Hylaeus duckei (Nlfken, 19()4)

Hylaeus enryscapus Förster, 1871

Hylaeus gibbus gibbus Saunders, 1850

Hylaeus gibbus confusus Nylander, 1852

Hylaeus gracilicornis (Morawitz, 1867)

Hylaeus hyalinatus Smith, 1842

Hylaeus leptocephalus (Morawitz, 1870)

Hylaeus lineolatus (Schenck, 1859)

Hylaeus moricei (Friese, 1898)

Hylaeus nigritus (Fabricius, 1798)

Hylaeus pectoralis Förster, 1871

Hylaeus pfankuchi (Alfken, 1919)

Hylaeus pictipes Nylander, 1852

Hylaeus punctatus Förster, 1871

Hylaeus punctulatissimus Smith, 1842

Hylaeus rinki (Gorski, 1852)

Hylaeus signatus (Panzer, 1798)

Hylaeus sinuatus (Schenck, 1853)

Hylaeus styriacus Förster, 1871

Hylaeus trinotatus (Pérez, 1895)

Hylaeus variegatus (Fabricius, 1798)

Colletes carinatus gallicus Radoszkovski, 1891

Colletes collaris Dours, 1872

Colletes cunicularius (Linné, 1761)

Colletes daviesanus Smith, 1846

Colletes fodiens (Geoffroy, 1785)

Colletes ligatus hylaeiformis Eversmann, 1852

Colletes inexpectatus Noskiewicz, 1936

Colletes lebedewi Noskiewicz, 1936

Colletes marginatus Smith, 1853

Colletes mlokossewiczi Radoszkowski, 1891

Colletes nasutus Smith, 1853

Colletes pallescens Noskiewicz, 1936

Colletes similis Schenck, 1853

Colletes succinctus (Linné, 1758)

\section{Andreninae}

Andrena aciculata Morawitz, 1886

Andrena aeneiventris Morawitz, 1872

Andrena agilissima (Scopoli, 1770)

Andrena alfkenella Perkins, 1914

Andrena angustior fulvata Stöckhert, 1930

Andrena apicata Smith, 1847

Andrena argentata Smith, 1844

Andrena atrata Friese, 1887

Andrena barbilabris (Kirby, 1802)
1
$20,26,27,29,30,32,33,34,35$

$20,26,27,29,30,32,33,34,35$

$20,26,27,29,30,32,33,34,35$ 34

$20,26,29,30,32,33,35$

$20,29,30,32,35$

$20,29,32,33,35$

$20,29,32,35$

20

$20,26,27,29,34$

$20,26,27,29,30,32,33,34,35$

20,35

$29,30,32,35$

$20,26,29,30,33,35$

20,35

$26,30,35$

$29,33,35$

20,35

$20,32,35$

20,35

$20,26,29,32,33,35$

29,35

$20,32,35$

$20,29,30,32,33,35$

$20,26,29,30,32,33,35$

$29,30,32,33,35$

20,35

$20,26,27,29,30,32,33,34,35$

35

9,21

$21,26,29,30,32,34,35$

$2 \mathrm{I}, 29,30,32,34,35$

$2 \mathrm{I}, 26,27,29,30,33,34,35$

$21,29,30,35$

$21,30,33,34,35$

$9,21,35$

$21,34,35$

29,35

$21,29,35$

$26,30,32,35$

$21,26,29,30,32,33,34,35$

21,35

35

$24,29,35$

$29,32,35$

$29,30,32,34,35$

$26,29,30,32,34,35$

$26,30,33$

$24,26,29,30,32,33,34,35$

24,35

$27,29,30,32,33,34,35$ 
Andrena bicolor Fabricius, 1775

Andrena bimaculata bimaculata (Kirby, 1802)

Andrena bimaculata morawitzi Thomson, 1872

Andrena bisulcata Morawitz, 1877

Andrena braunsiana Friese, 1887

Andrena bucephala Stephens, 1846

Andrena carbonaria (Linné, 1767)

Andrena chrysopus Pćrcz, 1903

Andrena chrysopyga Schenck, 1853

Andrena chrysosceles (Kirby, 1802)

Andrena cineraria (Linné, 1758)

Andrena combinata (Christ, 1791)

Andrena cordialis Morawitz, 1877

Andrena currana Warncke, 1965

Andrena denticulata (Kirby, 1802)

Andrena dorsalis Brullé, 1832

Andrena dorsata dorsata (Kirby, 1802)

Andrena dorsata propinqua Schenck, 1853

Andrena enslinella Stöckhert, 1924

Andrena falsifica Perkins, 1915

Andrena flavipes Panzer, 1799

Andrena florea Fabricius, 1793

Andrena floricola Eversmann, 1852

Andrena florivaga Eversmann, 1852

Andrena fulva (Müller, 1766)

Andrena fulvago (Christ, 1791)

Andrena fulvicornis Schenck, 1853

Andrena fulvida Schenck, 1853

Andrena fuscosa Erichson, 1835

Andrena gravida Imhoff, 1832

Andrena haemorrhoa (Fabricius, 1781)

Andrena hattorfiana (Fabricius, 1775)

Andrena helvola (Linné, 1758)

Andrena humilis Imhoff, 1832

Andrena impunctata Pérez, 1895

Andrena labilalis (Kirby, 1802)

Andrena labiata Fubricius, 1781

Andrena lagopus Latreille, 1809

Andrena lathyri Alfken, 1899

Andrena marginata Fabricius, 1776

Andrena minutula (Kirby, 1802)

Andrena minutuloides Perkins, 1914

Andrena mitis Schmiedeknecht, 1883

Andrena mocsaryi Schmiedeknecht, 1883

Andrena nana (Kirby, 1802)

Andrena nanula Nylander, 1848

Andrena nasuta Giraud, 1863

Andrena nigroaenea (Kirby, 1802)

Andrena nitida nitida (Müller, 1776)

Andrena nitida limata Smith, 1853

Andrena nitidiuscula Schenck, 1853

Andrena niveata Friese, 1887

Andrena nobilis Morawitz, 1874

Andrena numida hypopolia Schmiedeknecht, 1883

Andrena nychtemera Imhoff, 1866
$29,30,33,34,35$

$24,26,29,30,32,33,34,35$

30,35

$24,29,30,34,35$

24

$29,30,32,35$

$24,32,35$

35

$24,29,32,35$

32,35

$29,32,35$

29,35

$24,29,30,32,35$

$29,30,32,35$

$24,26,29,32,33,34,35$

$29,32,34,35$

$24,26,27,29,30,32,33,34,35$

$24,26,27,29,32,33,34,35$ 35

$26,27,29,30,32,33,35$

$26,29,30,32,33,34,35$

$29,30,32,35$

$29,34,35$

$29,30,35$

$29,30,32,33,34,35$

$29,34,35$

$29,30,32,35$

26,35

24,35

$24,29,30,32,33,34,35$

$24,26,27,29,30,32,33,34,35$

$24,29,30,32,34,35$

$29,30,32,33,34,35$

$26,29,32,34,35$

$24,29,30,32,35$

$24,29,32,34,35$

$26,29,30,32,33,34,35$

$29,30,34,35$

$27,29,30,32,33,35$

$24,29,30,33,34,35$

$26,29,30,32,33,34,35$

$24,26,29,30,32,33,34,35$

$30,32,34,35$

$29,30,35$

$29,30,34,35$

$29,30,35$

$24,29,33,35$

$24,30,32,35$

$29,30,32,33,34,35$

$24,29,30,32,33,34,35$

$24,26,29,30,32,33,34,35$

$27,29,32,35$

35

$24,29,35$

$24,26,29,30,35$ 
Andrena obsoleta spongiosa Warncke, 1967

Andrena ocreata (Christ, 1791)

Andrena oralis Morawitz, 1876

Andrena ovatula (Kirby, 1802)

Andrena pallitarsis Pérez, 1903

Andrena pandellei europaea Warncke, 1967

Andrena paucisquama Noskiewicz, 1924

Andrena pellucens Pérez, 1895

Andrena pillichi Noskiewicz, 1939

Andrena polita Smith, 1847

Andrena potentillae Panzer, 1809

Andrena praecox (Scopoli, 1763)

Andrena proxima (Kirby, I 802)

Andrena rosae Panzer, 1801

Andrena roseipes Alfken, 1933

Andrena rufula Schmiedeknecht, 1883

Andrena sabulosa sabulosa (Scopoli, 1763)

Andrena sabulosa trimmerana (Kirby, 1802)

Andrena saxonica Stöckhert, 1935

Andrena schencki Morawitz, 1866

Andrena schlettereri Friese, 1896

Andrena scita Eversmann, 1852

Andrena seminuda Friese, 1896

Andrena sericata Imhoff, 1866

Andrena simontomyella Noskiewicz, 1939

Andrena spreta pusilla Pérez, 1903

Andrena strohmella Stöckhert, 1928

Andrena subopaca Nylander, 1848

Andrena suerinensis Friese, 1884

Andrena susterai Alfken, 1914

Andrena symphyti Schmiedeknecht, 1883

Andrena taraxaci Giraud, 1861

Andrena thoracica (Fabricius, 1775)

Andrena tibialis (Kirby, 1802)

Andrena tridentata (Kirby, 1802)

Andrena truncatilabris Morawitz, 1877

Andrena tscheki Morawitz, 1872

Andrena vaga Panzer, 1799

Andrena variabilis Smith, 1853

Andrena varians (Kirby, 1802)

Andrena ventralis Imhoff, 1832

Andrena ventricosa Dours, 1873

Andrena viridescens Viereck, 1916

Andrena wilkella (Kirby, 1802)

Panurgus calcaratus (Scopoli, 1763)

Panurginus labiatus (Eversmann, 1852)

Melitturga clavicomis (Latreille, 1806)

Camptopoeum frontale (Fabricius, 1804)

\section{Halictinae}

Halictus (Halictus) asperulus Pérez, 1895

Halictus (H.) brunnescens (Eversmann, 1852)

Halictus (H.) eurygnathus Blüthgen, 1936

Halictus (H) fulvipes (Klug, 1817)

Halictus $(H$.$) langobardicus Blüthgen, 1944$
32,35

35

35

$10427,29,30,32,33,34,35$

$7 \quad 24,29,34,35$

$17 \quad 33,35$

I $29,32,33,35$

I 24

l 24,35

$24 \quad 29,34,35$

$3 \quad 35$

$55 \quad 24,26,27,29,30,32,33,35$

$73 \quad 29,30,32,34,35$

$57 \quad 24,29,30,32,33,34,35$

$2 \quad 35$

624,35

$41 \quad 29,30,32,33,35$

$8 \quad 29,35$

$18 \quad 29,32,35$

$21 \quad 29,30,32,34,35$

$21 \quad 29,35$

$2 \quad 24,35$

$40 \quad 29,30,32,34,35$

$24 \quad 29,30,32,35$

$52 \quad 29,30,32,34,35$

$10 \quad 29,30,35$

430,35

$6924,26,27,29,30,32,33,34,35$

$20 \quad 24,27,29,32,35$

$67 \quad 29,30,32,33,34,35$

$51 \quad 29,30,32,33,34,35$

$72 \quad 29,30,32,33,34,35$

$2 \quad 24,29,35$

$45 \quad 24,26,27,29,32,22,34,35$

$1 \quad 35$

1134,35

$1429,30,34,35$

$53 \quad 26,29,30,32,33,34,35$

124,35

$2929,30,32,35$

$33 \quad 26,29,30,32,34,35$

$7 \quad 29,35$

$6127,29,30,32,33,34,35$

124

$67 \quad 26,29,30,32,33,34,35$

$1429,30,35$

$5 \quad 29,30,35$

135

$3 \quad 35$

$4 \quad 29,35$

$1 \quad 35$

$6329,30,32,34,35$ 
Halictus (H.) maculatus Smith, 1848

Halictus (H.) quadricinctus (Fabricius, I776)

Halictus (H.) rubicundus (Christ, 1791)

Halictus (H.) sajói Blüthgen, 1923

Halictus (H.) scabiosae (Rossi, 1790) I

Halictus (H.) sexcinctus (Fabricius, 1775)

Halictus (H.) simplex Blüthgen, 1923

Halictus (Seladonia) confusus perkinsi Blüthgen, 1925

Halictus (S.) kessleri Bramson, 1879

Halictus (S.) leucaheneus arenosus Ebmer, 1976

Halictus (S.) seladonius (Fabricius, 1794)

Halictus (S.) semitectus Morawitz, 1873

Halictus (S.) smaragdulus Vachal, 1895

Halictus (S.) subauratus (Rossi, 1792)

Halictus (S.) tumulorum (Linné, 1758)

Halictus (Vestitohalictis) tectus Radoszkowski, 1875

Lasioglossum (Lasioglossum) costulatum

(Kriechbaumer, 1873)

Lasioglossum (L.) discum (Smith, 1853)

Lasioglossum (L.) kussariense (Blüthgen, 1924)

Lasioglossum (L.) laevigatum (Kirby, 1802)

Lasioglossum (L.) laterale (Brullé, 1832)

Lasioglossum (L.) lativentre (Schenck, 1853)

Lasioglossum (L.) leucozonium (Schrank, 1781)

Lasioglossum (L.) majus (Nylander, 1852)

Lasioglossum (L.) pallens (Brullé, 1832)

Lasioglossum (L.) pseudocaspicum (Blüthgen, 1923)

Lasioglossum (L.) quadrinotatum (Kirby, 1802)

Lasioglossum (L.) sexnotatum (Kirby, 1802)

Lasioglossum (L.) subfasciatum (Imhoff, 1832)

Lasioglossum (L.) xanthopum (Kirby, 1802)

Lasioglossum (L.) zonulum (Smith, 1848)

Lasioglossum (Evylaeus) aeratum (Kirby, 1802)

Lasioglossum (E.) albipes (Fabricius, 1781)

Lasioglossum (E) angusticeps (Perkins, 1895)

Lasioglossum (E.) bluethgeni Ebmer, 1971

Lasioglossum (E.) brevicorne brevicorne (Schenck, 1868) 37

Lasioglossum (E.) brevicorne aciculatum (Blüthgen, 1930) 7

Lasioglossum (E.) buccale (Pérez, 1903)

Lasioglossum (E.) calceatum (Scopoli, 1763)

Lasioglossum (E.) clypeare (Schenck, 1853)

Lasioglossum (E.) convexiusculum (Schenck, 1853)

Lasioglossum (E.) crassepunctatum (Blüthgen, 1923)

Lasioglossum (E.) damascenum (Pérez, 1911)

Lasioglossum (E.) elegans (Lepeletier, 1841)

Lasioglossum (E.) euboeense (Strand, 1909)

Lasioglossum (E.) fulpicorne (Kirby, 1802)

Lasioglossum (E.) glabriusculum (Morawitz, 1872)

Lasioglossum (E.) griseolum (Morawitz, 1872)

Lasioglossum (E.) intermedium (Schenck, 1868)

Lasioglossum (E.) interruptum (Schenck, 1868)

Lasioglossum (E.) laeve (Kirby, 1802)

Lasioglossum (E.) laticeps (Schenck, 1868)

Lasioglossum (E.) leucopum (Kirby, 1802)

Lasioglossum (E.) limbellum (Morawitz, 1876)
$27,29,30,32,33,34,35$

$29,33,35$

$26,27,29,30,32,33,34,35$

29,35

$29,32,35$

$27,29,30,32,33,34,35$

$10,29,30,32,33,34,35$

$26,27,29,30,32,34,35$

29,35

$26,27,29,30,33,35$

$26,29,30,32,33,34,35$

$10,26,30,32,33,34,35$

10,35

$26,29,30,32,33,34,35$

$29,30,32,34,35$

35

$29,30,2,35$

$29,32,34,35$

29

$29,30,32,34,35$

$10,29,30,32,33,34,35$

$26,29,30,34,35$

$26,29,30,32,33,34,35$

$27,29,30,32,33,34,35$

$27,29,30,32,33,34,36$

29

29,35

$27,29,30,32,33,34,35$

29,35

$29,32,35$

$27,29,30,32,34,35$

$10,26,27,30,32,33,35$

$27,29,30,32,33,34,35$

35

$27,29,32,34,35$

$26,29,30,32,33,34,35$

$26,33,34,35$

$29,30,32,34,35$

$27,29,30,32,33,34,35$

$29,30,32,34,35$

$29,32,34,35$

35

35

10,35

$27,30,32,35$

$10,29,30,32,34,35$

35

35

$29,30,33,34,35$

35

$27,29,30,32,33,34,35$

10,35

35 
Lasioglossum (E.) lineare (Schenck, 1868)

Lasioglossum (E.) lucidulum (Schenck, 1859)

Lasioglossum (E.) malachurum (Kirby, 1802)

Lasioglossum (E.) marginatum (Brullé, 1832)

Lasioglossum (E.) marginellum (Schenck, 1853)

Lasioglossum (E.) mesosclerum (Pérez, 1903)

Lasioglossum (E.) minutissimum (Kirby, 1802)

Lasioglossum (E.) minutulum (Schenck, 1853)

Lasioglossum (E.) morio (Fabricius, 1793)

Lasioglossum (E.) nigripes (Lepeletier, 1841)

Lasioglossum (E.) nitidulum (Fabricius, 1804)

Lasioglossum (E.) nitidiusculum (Kirby, 1802)

Lasioglossum (E.) obscuratum acerbum (Wancke, 1975)

Lasioglossum (E.) pauxillum (Schenck, 1853)

Lasioglossum (E.) parrulum (Schenck, 1853)

Lasioglossum (E.) politum (Schenck, 1853)

Lasioglossum (E.) punctatissimum (Schenck, 1853)

Lasioglossum (E.) puncticolle (Morawiť, 1872)

Lasioglossum (E.) pygmaeum (Schenck, 1853)

Lasioglossum (E.) quadrinotatulum (Schenck, 1853)

Lasioglossum (E.) quadrisignatum (Schenck, 1853)

Lasioglossum (E.) semilucens (Alfken, 1814)

Lasioglossum (E.) setulosum (Strand, 1909)

Lasioglossum (E.) sexstrigatum (Schenck, 1868)

Lasioglossum (E.) subhirtum (Lepeletier, 1841)

Lasioglossum (E.) tarsatum (Schenck, 1868)

Lasioglossum (E.) trichopygum (Blüthgen, 1923)

Lasioglossum (E.) truncaticolle (Morawitz, 1878)

Lasioglossum (E.) pillosulum (Kirby, 1802)

Sphecodes albilabris (Fabricius, 1793)

Sphecodes alternatus Smith, 1853

Sphecodes crassus Thomson, 1870

Sphecodes cristatus Hagens, 1882

Sphecodes croaticus Meyer, 1922

Sphecodes ephippius (Linné, 1767)

Sphecodes ferruginatus Hagens, 1882

Sphecodes geofrellus (Kirby, 1802)

Sphecodes gibbus (Linné, 1758)

Sphecodes intermedius Blüthgen, 1923

Sphecodes longulus Hagens, 1882

Sphecodes majalis Pérez, 1903

Sphecodes miniatus Hagens, 1882

Sphecodes monilicomis (Kirby, 1802)

Sphecodes niger Hagens, 1874

Sphecodes pellucidus Smith, 1845

Sphecodes pseudofasciatus Blüthgen, 1924

Sphecodes puncticeps Thomson, 1870

Sphecodes reticulatus Thomson, 1870

Sphecodes ruficrus rubicundus Hagens, 1875

Sphecodes rufiventris (Panzer, 1798)

Sphecodes scabricollis Wesmael, 1865

Sphecodes spinulosus Hagens, 1875

Nomioides minutissimus (Rossi, 1790)

Nomioides variegatus (Olivier, 1789)

Pseudapis diversipes (Latreille, 1806)
$29,34,35$

$26,27,29,30,32,33,34,35$

$29,30,33,34,35$

$29,30,32,33,34,35$

$10,29,34,35$

$10,29,34,35$

$10,29,30,32,33,34,35$

35

$29,30,32,33,34,35$

$29,32,33,34,35$

$29,30,32,33,34,35$

$10,29,30,32,33,34,35$

35

$27,29,30,32,33,34,35$

$29,34,35$

$27,29,30,32,33,34,35$

$26,29,30,32,33,34,35$

$26,27,29,34,35$

$10,29,35$

$10,29,30,32,33,34,35$

29

$29,30,33,34,35$

35

$26,27,29,30,32,33,34,35$

10,35

$10,27,32,35$

$29,30,34,35$

29

$10,26,29,30,32,33,34,35$

$27,29,30,32,33,34,35$

$23,29,34,35$

$27,29,30,33,35$

$23,27,30,33,34,35$

$23,26,29,32,35$

$26,29,30,32,33,34,35$

$23,29,30,35$

27,35

$27,29,30,32,34,35$

$10,23,35$

$10,23,29,30,32,33,34,35$

$29,30,35$

$23,29,32,33,34,35$

$29,30,32,33,34,35$

$29,32,34,35$

$23,29,3,32,33,34,35$

$29,30,35$

$23,29,30,32,33,34,35$

$23,30,35$

29,35

$23,29,30,32,33,34,35$

$10,23,29,32,35$

35

$10,26,27,2,30,32,33,34,35$

$26,29,30,32,33,34,35$

35 
Pseudapis femoralis (Pallas, 1773)

Rophites algirus trispinosus Pérez, 1903

Rophites hartmanni Friese, 1902

Rophites quinquespinosus Spinola, 1818

Rhophitoides canus (Eversmann, 1852)

Systropha curvicornis (Scopoli, 1770)

Systropha planidens Giraud, 1861

\section{Melittinae}

Melitta dimidiata Morawitz, 1876

Melitta haemorrhoidalis (Fabricius, 1775)

Melitta leporina (Panzer, 1799)

Melitta nigricans Alfken, 1905

Melitta tricincta Kirby, 1802

Macropis frivaldszkyi Mocsáry, 1878

Macropis fulvipes (Fabricius, I 804)

Macropis labiata (Fabricius, 1804)

Dasypoda hirtipes (Fabricius, 1793)

\section{Megachilinae}

Lithurgus chrysurus Fonscolombe, 1834

Lithurgus cornutus fuscipennis Lepeletier, 1841

Trachusa byssina (Panzer, 1798)

Anthidium cingulatum Latreille, 1809

Anthidium florentinum (Fabricius, 1775)

Anthidium laterale Latreille, 1809

Anthidium lituratum (Panzer, 1801)

Anthidium manicatum (Linné, 1758)

Anthidium oblongatum (Illiger, 1806)

Anthidium punctatum Latreille, 1809

Anthidium septemspinosum Lepeletier, 1841

Anthidium strigatum (Panzer, 1805)

Anthidium tenellum Mocsáry, 1879

Stelis breviuscula (Nylander, 1848)

Stelis jugae Noskiewicz, 1962

Stelis minuta Lepeletier et Serville, 1825

Stelis odontopyga Noskiewicz, 1925

Stelis ornatula (Klug, 1807)

Stelis phaeoptera (Kirby, I 802)

Stelis punctulatissima (Kirby, 1802)

Stelis signata (Latreille, 1809)

Dioxys cincta (Jurine, 1807)

Dioxys pannonica Mocsáry, 1877

Dioxys tridentata (Nylander, 1848)

Chelostoma appendiculatum (Morawitz, 1871)

Chelostoma campanularum (Kirby, 1802)

Chelostoma distinctum Stöckhert, 1929

Chelostoma florisomne (Linné, 1758)

Chelostoma fuliginosum (Panzer, 1798)

Chelostoma handlirschi Schletterer, 1889

Chelostoma ventrale Schletterer, 1889

Heriades crenulatus Nylander, 1856

Heriades rubicolus Pérez, 1890

Heriades truncorum (Linné, 1758)

Hoplitis acuticomis (Dufour et Perris, 1840)
1

4

31

33

31

46

3

4

4

43

36

22

1

11

27

57

25

17

11

I

16

2

56

54

70

14

3

37

3

30

10

5

3

12

1

18

5

1

1

2

50

42

25

50

31

2

25

53

1

9

7
35

29,35

$29,30,32,34,35$

$29,30,34,35$

29,35

$16,29,30,32,33,34,35$

29,35

29,35

29

$16,29,32,34,35$

$29,30,32,33,34,35$

$16,29,30,35$

29

$26,29,30,32,34,35$

$16,26,29,30,32,33,35$

$16,26,29,30,32,33,34,35$

$26,29,32,33,34,35$

$29,32,33,34,35$

$25,29,35$

15

$15,29,34,35$

15,35

$15,26,27,29,30,32,33,34,35$

$15,29,30,32,33,34,35$

I5, $29,30,32,33,34,35$

$25,29,30,32,35$

32,33

$15,16,27,29,30,32,33,34,35$

15,35

$29,30,32,33,34,35$

35

$25,29,32,34,35$

29,35

$25,26,29,30,32,35$

33

$29,30,32,35$

$25,30,32,33,34,35$

8

25,29

35

$8,29,30,32,34,35$

$29,30,32,33,34,35$

$8,26,29,30,32,33,34,35$

$29,30,32,33,34,35$

$29,30,32,34,35$

$29,30,33,34,35$

$26,29,30,32,33,34,35$

35

$26,27,29,30,32,33,34,35$

$29,32,35$ 
Hoplitis adunca (Panzer, 1798)

Hoplitis anthocopoides (Schenck, 1853)

Hoplitis claviventris (Thomson, 1872)

Hoplitis leucomelaena (Kirby, 1802)

Hoplitis manicata (Morice, 1901)

Hoplitis ravouxi (Pérez, 1902)

Hoplitis rufohirta (Latreille, 1811)

Hoplitis tenuispina (Alfken, 1937)

Hoplitis tridentata (Dufour et Perris, 1840)

Anthocopa andrenoides (Spinola, 1808)

Anthocopa bidentata (Morawitz, 1876)

Anthocopa laevifrons (Morawitz, 1872)

Anthocopa ligurica (Morawitz, 1868)

Anthocopa mocsaryi (Friese, 1895)

Anthocopa papaveris (Latreille, 1799)

Anthocopa spinulosa (Kirby, 1802)

Anthocopa tergestensis (Ducke, 1897)

Osmia bicolor (Schrank, 1781)

Osmia coerulescens (Linné, 1758)

Osmia cerinthidis Morawitz, 1876

Osmia cornuta (Latreille, 1805)

Osmia fulviventris (Panzer, 1798)

Osmia gallarum Spinola, 1808

Osmia melanogaster Spinola, 1808

Osmia pilicornis Smith, 1846

Osmia rufa globosa (Scopoli, 1763)

Osmia tunensis aurulenta (Panzer, 1799)

Osmia ventralis (Panzer, 1798)

Metallinella brevicomis (Fabricius, 1798)

Chalicodoma ericetorum (Lepeletier, 1841)

Chalicodoma parietinum (Geoffroy, 1785)

Megachile albisecta (Klug, 1817)

Megachile apicalis Spinola, 1808

Megachile argentata (Fabricius, 1793)

Megachile bombycina Radoszkowski, 1874

Megachile centuncularis (Linné, 1758)

Megachile circumcincta (Kirby, 1802)

Megachile deceptoria Pérez, 1890

Megachile genalis Morawitz, 1880

Megachile lagopoda (Linné, 1761)

Megachile ligniseca (Kirby, 1802)

Megachile maritima (Kirby, 1802)

Megachile melanopyga Costa, 1863

Megachile octosignata Nylander, 1852

Megachile pilicrus Morawitz, 1877

Megachile pilidens Alfken, 1924

Megachile rotundata (Fabricius, 1787)

Megachile rubrimana Morawitz, 1894

Megachile versicolor Smith, 1844

Megachile willougbiella (Kirby, 1802)

Coelioxys afra Lepeletier, 1841

Coelioxys aurolimbata Förster, 1853

Coelioxys brevis Eversmann, 1852

Coelioxys conoidea (Illiger, 1806)

Coelioxys elongata Lepeletier, 1841
$29,32,35$

$26,27,29,30,32,33,34,35$

$26,29,30,32,33,34,35$

$29,30,33,35$

29

$29,32,35$

29,35

$25,29,34,35$

29,35

$26,29,32,34,35$

25,29

25,29

35

$8,29,35$

$29,30,32,33,34,35$

$25,29,35$

$26,27,30,32,33,34,35$

$26,29,30,32,33,34,35$

$29,33,35$

$29,32,33,35$

$27,29,30,35$

$25,29,30,35$

$27,29,35$

25,29

$26,29,30,31,33,34,35$

$26,29,30,31,33,34,35$

30,35

30,35

$29,30,33,34,35$

35

35

$29,31,35$

$29,30,31,33,34,35$

8,35

$26,27,29,30,31,33,34,35$

$26,29,30,33,35$

29,35

$25,29,35$

29,35

$29,30,31,33,35$

$26,29,30,31,33,35$

29,35

29,31

$26,29,30,31,34,35$

$29,31,34,35$

$26,29,30,31,33,34,35$

29

$29,30,31,35$

$26,27,29,31,34,35$

$29,34,35$

$29,32,34,35$

$30,34,35$

$26,30,32,34,35$

$29,32,34,35$ 
Coelioxys inermis (Kirby, 1802)

Coelioxys mandibularis Nylander, 1848

Coelioxys obtusa Pérez, 1884

Coelioxys polycentris Förster, 1853

Coelioxys quadridentata (Linné, 1761)

Coelioxys rufescens Lepeletier, 1825

Coelioxys rufocaudata Lepeletier, 1841

\section{Anthophorinae}

Nomada alboguttata Herrich-Schaeffer, 1839

Nomada argentata Herrich-Schaeffer, 1839

Nomada armata Herrich-Schaeffer, 1839

Nomada atroscutellaris Strand, 1921

Nomada baccata hrubanti Balthasar, 1958

Nomada basalis Herrich-Schaeffer, 1839

Nomada bifasciata fucata Panzer, 1798

Nomada bifasciata lepeletieri Pérez, 1884

Nomada bifida Thomson, 1872

Nomada bispinosa Mocsáry, 1883

Nomada bluethgeni Stöckhert, 1943

Nomada blepharipes Schmiedeknecht, 1882

Nomada braunsiana Schmiedeknecht, 1882

Nomada calimorpha Schmiedeknecht, 1882

Nomada castellana Dusmet, 1913

Nomada chrysopyga Morawitz, 1871

Nomada conjungens Herrich-Schaeffer, 1839

Nomada distinguenda Morawitz, 1874

Nomada emarginata Morawitz, 1877

Nomada fabriciana (Linné, 1767)

Nomada facilis Schwarz, 1967

Nomada ferruginata (Linné, 1767)

Nomada flava Panzer, 1798

Nomada flaroguttata (Kirby, 1802)

Nomada flavopicta (Kirby, 1802)

Nomada fulvicomis Fabricius, 1793

Nomada fulvicornis schmiedeknechti

Schmiedeknecht, 1882

Nomada furva Panzer, 1798

Nomada fuscicornis Nylander, 1848

Nomada guttulata Schenck, 1859

Nomada hirtipes Pérez, 1884

Nomada hungarica Dalla Torre et Friese

Nomada incisa Schmiedeknecht, 1882

Nomada integra Brullé, 1832

Nomada kohli Schmiedeknecht, 1882

Nomada lathburiana (Kirby, 1802)

Nomada leucophthalma (Kirby, 1802)

Nomada marshamella (Kirby, 1802)

Nomada melanopyga Schmiedeknecht, 1882

Nomada mutabilis Morawitz, 1870

Nomada panzeri Lepeletier, 1841

Nomada panzeri glabella Thomson, 1870

Nomada panzeri hybrida Schmiedeknecht, 1882

Nomada platythorax Schwarz, 1981

Nomada pleurosticta Herrich-Schaeffer, 1839
49

7

4 .

3

21

19

19

28

7

18

26

I

7

59

73

39

4

32

1

2

1

15

1
$26,29,30,32,33,34,35$

$29,30,35$

29,35

$8,25,29,35$

$29,30,32,33,34,35$

$26,29,30,32,34,35$

$29,34,35$

$27,29,30,34,35$

$26,29,35$

$29,30,32,35$

$29,30,32,34,35$

26

$22,29,35$

$27,29,30,32,34,35$

$29,30,32,35$

$29,30,32,34,35$

29

$29,30,32,34,35$

22,35

35

35

$22,29,35$

35

$29,30,32,34,35$

$26,27,29,30,32,34,35$

35

$29,30,34,35$

29

$26,29,30,35$

$29,30,32,34,35$

$29,30,32,34,35$

$22,29,34,35$

$22,29,34,35$

22,35

$26,29,32,34,35$

$26,29,35$

22,35

29

22,35

35

29

30

$29,30,32,35$

$30,33,34,35$

$32,34,35$

35

29

$29,30,32,33,34,35$

$29,30,32,34,35$

$32,33,35$

35

$29,34,35$ 
Nomada posthuma Blüthgen, 1949

Nomada henana Morawitz, 1872

Nomada rostrata Herrich-Schaeffer, 1839

Nomada rufipes Fabricius, 1793

Nomada sexfasciata Panzer, 1799

Nomada sheppardana (Kirby, 1802)

Nomada sheppardana minuscula Noskiewitz, 1930

Nomada signata Jurine, 1807

Nomada stigma Fabricius, 1804

Nomada striata Fabricius, 1793

Nomada succincta Panzer, 1798

Nomada symphyti Stöckhert, 1930

Nomada trapeziformis Schmiedeknecht, 1882

Nomada tridentirostris Dours, 1837

Nomada trispinosa Schmiedeknecht, 1882

Nomada verna Schmiedeknecht, 1882

Nomada villosa Thomson, 1870

Nomada zonata Panzer, 1798

Ammobates punctatus (Fabricius, 1804)

Pasites maculatus Jurine, 1807

Parammobatodes minutus (Mocsáry, 1878)

Ammobatoides abdominalis (Eversmann, 1852)

Biastes breviconis (Panzer, 1798)

Biastes emarginatus (Schenck, 1853)

Epeolus cruciger (Panzer, 1799)

Epeolus tristis Smith, 1854

Epeolus variegatus (Linné, 1758)

Epeoloides coecutiens (Fabricius, 1775)

Tetralonia acutangula Morawitz, 1877

Tetralonia armeniaca Morawitz, 1878

Tetralonia dentata (Klug, 1835)

Tetralonia hungarica (Friese, 1895)

Tetralonia macroglossa Illiger, 1806

Tetralonia nana Morawitz, 1873

Tetralonia ruficornis (Fabricius, 1804)

Tetralonia salicariae (Lepeletier, 1841)

Tetralonia tricincta (Erichson, 1835)

Eucera chrysopyga Pérez, 1879

Eucera cinerea Lepeletier, 1841

Eucera clypeata Erichson, 1835

Eucera intermuta Baer, 1850

Eucera longricomis (Linné, 1758)

Eucera nitidiventris Mocsáry, 1879

Eucera parnula Friese, 1895

Eucera parviconis Mocsáry, 1878

Eucera seminuda Brullé, 1832

Eucera taurica Morawitz, 1870

Eucera tuberculata (Fabricius, 1793)

Clisodon furcatus (Panzer, 1798)

Amegilla albigena (Lepeletier, 1841)

Amegilla garrula (Rossi, 1790)

Amegilla quadrifasciata (Villers, 1789)

Amegilla salviae (Morawitz, 1856)

Anthophora acervorum (Linné, 1758)

Anthophora aestivalis (Panzer, 1801)
27,35

$29,33,35$

22,29

$29,32,33,35$

$27,29,34,35$

$29,30,34,35$

$27,29,30,35$

22,35

$\begin{array}{ll}10 & 35 \\ 2 & 29,35\end{array}$

$45 \quad 27,29,30,32,33,34,35$

$46 \quad 29,30,32,34,35$

229,32

129

$47 \quad 29,34,35$

229

$629,30,35$

$1929,30,35$

$8 \quad 26,30,32,33,34,35$

222,35

135

$3 \quad 25,29$

$37 \quad 19,29,30,32,33,34,35$

$7 \quad 25,29,35$

$10 \quad 26,29,30,32,35$

$31 \quad 19,29,30,33,34,35$

$30 \quad 26,29,30,32,33,34,35$

II $7,19,26,29,30,32,33,35$

129

$1 \quad 13,35$

$1313,29,30,33,34,35$

$6 \quad 13,29,35$

$5213,29,32,32,33,34,35$

$22 \quad 13,39,32,35$

$14 \quad 13,39,35$

$31 \quad 13,29,30,32,33,34,35$

$3 \quad \mathrm{I} 3,29,35$

$22 \quad 13,29,35$

313,35

$11 \quad 13,29,35$

$37 \quad 13,29,32,33,35$

$22 \quad 13,29,35$

$13 \quad 13,29,35$

$9 \quad 29,34,35$

213,35

I3 13, 29, 35

$1 \quad 13,35$

$108 \quad 13,26,27,29,30,32,33,34,35$

$34 \quad 18,29,30,32,33,34,35$

$4 \quad 18,29,35$

$6 \quad 29,34,35$

318,35

$48 \quad 18,26,29,30,32,33,34,35$

$73 \quad 18,29,30,32,33,34,35$ 
Anthophora crinipes Smith, 1854

Anthophora parietina (Fabricius, 1793)

Anthophora pubescens (Fabricius, 178I)

Anthophora quadrimaculata (Panzer, 1806)

Anthophora retusa (Linné, 1758)

Heliophila bimaculata (Panzer, 1798)

Melecta luctuosa (Scopoli, 1770)

Melecta punctata (Fabricius, 1775)

Thyreus historionicus (Pérez, 1883)

Thyreus orbatus (Lepeletier, 1841)

Thyreus ramosus (Lepeletier, 1841)

Thyreus truncatus (Pérez, 1883)

Ceratina acuta Friese, 1896

Ceratina callosa (Fabricius, 1794)

Ceratina cucurbitina (Rossi, 1792)

Ceratina cyanea (Kirby, 1802)

Ceratina gravidula Gerstaecker, 1869

Ceratina nigrolabiata Friese, 1896

Ceratina nigroaenea Gerstaecker, 1869

Xylocopa iris (Christ, 1791)

Xylocopa valga Gerstaecker, 1872

Xylocopa violacea (Linné, 1758)

\section{Apinae}

Bombus argillaceus (Scopoli, 1763)

Bombus confusus Schenck, 1859

Bombus haematurus Kriehbaumer, 1870

Bombus hortorum (Linné, 1761)

Bombus humilis Illiger, 1806

Bombus hypnorum ericetorum (Panzer, 1801 )

Bombus laesus mocsaryi Kriechbaumer, 1877

Bombus lapidarius (Linné, 1758)

Bombus muscorum (Linné, 1758)

Bombus paradoxus Dalla Torre, 1882

Bombus pascuorum collium (Scopoli, 1763)

Bombus pomorum (Panzer, 1805)

Bombus pratorum (Linné, 1761)

Bombus ruderarius (Müller, I776)

Bombus ruderatus eurynotus Dalla Torre, 1882

Bombus subterraneus nemorum (Scopoli, 1763)

Bombus sylvarum plumosus (Christ, 1791)

Bombus terrestris (Linné, 1758)

Psithyrus barbutellus (Kirby, 1802)

Psithyrus campestris (Panzer, 1801)

Psithyrus rupestris (Fabricius, 1793)

Psithyrus vestalis (Geoffroy, 1785)
$18,29,30,32,33,34,35$

$18,29,35$

$18,29,35$

$18,29,35$

$18,29,33,35$

$18,26,27,30,32,33,35$

$18,29,30,32,33,34,35$ 29

$18,29,33,35$

$18,29,30,34,35$

18,35

$19,29,35$

$19,26,27,29,30,32,33,34,35$

$29,30,32,34,35$

$19,26,27,29,32,33,34,35$

34

34

$19,29,35$

19

$19,26,29,30,32,33,34,35$

$19,29,30,32,33,35$

$11,25,29,35$

I 1, 25, 29, 35

$32,33,34$

$11,26,29,30,32,34,35$

$11,26,29,30,32,35$

$29,30,32,35$

I 1,35

$11,26,29,30,32,33,34,35$

$11,33,35$

$11,25,26,29,35$

$11,26,29,30,32,33,34,35$

11,35

$29,30,35$

$11,29,30,32,33,34,35$

11,35

11

$11,29,32,33,34,35$

$11,26,29,30,32,33,34,35$

$19,29,32,35$

$19,29,32,35$

19, 35

$19,29,32,35$ 


\section{Irodalom}

1. BAjARı, E. 1956: Törösdarázs alkatúak. - Scolioidea. - in: Magyarország Állatvilága (Fauna Hungariae), XIII/3.: 1-35.

2. BAIÁRI, E. 1957: Kaparódarázs alkatúak I. - Sphecoidea I. - In: Magyarország Állatvilága (Fauna Hungariae), XIII/7.: 1-117.

3. MóczÁR, L. 1956: Pókölódarázs alkatúak. - Pompiloidea. - In: Magyarország Állatvilága (Fauna Hungariae), XIII/5.: 1-76.

4. MócZÁR, L. 1959: Kaparódarázs alkatúak II. - Sphecoidea II. - In: Magyarország Állatvilága (Fauna Hungariae), XIII/8.: 1-87.

5. MóczÁr, L. 1967: Fémdarázs alkatúak. - Chrysidoidea. - In: Magyarország Állatvilága (Fauna Hungariae), XIII/2.: 1-1 I8.

6. MóczÁR, L. 1995: Redôsszárnyúdarázs-szerúek. - Vespoidea. - In: Magyarország Állatvilága (Fauna Hungariae), XIII/B./6.: 1-181.

7. MóczÁr, M. 1957: Méhfélék.- Apidae. - In: Magyarország Állatvilága (Fauna Hungariae), XIII/I3.: I-75.

8. MóczÁr, M. 1958: Múvészméhek. - Megachilidae. - In: Magyarország Állatvilága (Fauna Hungariae), XIIV/12.: 1-78.

9. MóczÁR, M. 1960: Ốsméhek - Földiméhek. - Colletidae - Melittidae. - In: Magyarország Állatvilága (Fauna Hungariae), XIII/9:: 1-64.

10. MóczÁr, M. 1967: Karcsúméhek. - Halictidae. - In: Magyarország Állatvilága (Fauna Hungariae), XIrI/9.: 1-116.

11. MóczÁr, M. 1953: A dongóméhek (Bombus Latr.) faunakatalógusa (Cat. Hym. IV.). - Fol. ent. hung. 6: 197-228.

I2. BajÁrı, E. - MóczÁr, L. 1954: A Methocidac, Myrmosidac és Multillidae családok faunakatalógusa (Cat. Hym. V.). - Fol. ent. hung. 7:61-78.

13. MóczÁr, M. 1955: Az Eucera Latr., Tetralonia Spin., Melitturga Latr. és az Ammobatoides Rad. nemzetségek faunakatalógusa (Cat. Hym. VII.). - Fol. Ent. Hung. 8: 111-129.

14. BAJÁrı, E. 1956: A csomósdarazsak (Cerceris Latr.) faunakatalógusa (Cat. Hym. VIII.). - Fol. ent. hung. 9:79-88.

15. MóCZÁr, M: 1956: A pelyhesméhek (Anthidium Fabr.) faunakatalógusa és etológiai adatai (Cat. Hym. IX.). - Fol. ent. hung. 9: 211-222.

16. MÓCZÁr, M. 1957: $\Lambda$ hazai földiméh-félék (Melittidae) faunakatalógusa és etológiai adatai (Cat. Hym. XI.). - Fol. ent. hung. 10: 459-471.

17. Móczér, L. 1958: ^ Crabroninae (Fam.: Sphecidae) alcsalád faunakatalógusa (Cat. Hym. XIII.). - Fol. ent. hung. 11: 189-216.

18. Móczír, M. 1958: A bundásméhek (Anthophora Latr.) és fészekélősködőik, a gyász és foltosméhek (Melecta Latr., Crocisa Latr.) revíziója, faunakatalógusa és etológiai adatai (Cat. Hym. XIV.). - Fol. ent. hung. 11: 403-421.

19. MóczÁR, M. 1959: Az Epeolus Latr., a Ceratina Latr. és a Psythirus Lep. nemek (Fam.: Apidae) faunakatalógusa és etológiai adatai (Cat. Hym. XVI.). - Fol. Ent. Hung., 12: 461-480.

20. MócZÁr, M. 1961: A Kárpát-medence ősméheinek - Colletidae - revíziója, faunakatalógusa és etológiai adatai I., Álarcosméhek - Prosopis F. (Cat. Hym. XVIII.). - Fol. ent. hung. 14:143-161.

21. MócZÁR, M. 1961b. A Kárpát-medence ôsméheinek - Colletidea - revíziója, faunakatalógusa és etológiai adatai II., Selyemméhek. Colletes Latr. (Cat. Hym. XIX.). - Fol. ent. hung. 14: 403-411.

22. MóCZÁr, L. - SCHWARZ, M. 1968: A Nomada-, Ammobates-, Pasites- és Parammobatodes nemek faunakatalógusa (Cat. Hym. XXIII.). - Fol. ent. hung. 21:339-360.

23. MóczÁr, L. - SCHWArz, M. 1970: A Sphecodes Latr. fajok faunakatalógusa (Cat. Hym. XXIV.). Fol. ent. hung. 23: 209-219.

24. Móczér, L. ET WarnCKe, K. (1972): Faunenkatalog der Gattung Andrena Fabricius (Cat. Hym., XXVI.). Acta Biol. Szeged 18:185-221.

25. Józan, Zs. 1971. Néhány adat Dél-Dunántúl méhalkatú (Hym., Apoidea) faunájához. - Folia ent. hung. 24: 109-117. 
26. JóZAN, Zs. I983: A Barcsi borókás fullánkos (Hymenoptera, Aculeata) faunája, I. - Dunántúli Dolgozatok Természettudományi Sorozat 3:89-111.

27. JózAN, Zs. 1985a: A Barcsi borókás fullánkos (Hymenoptera, Aculeata) faunája, Il. - Dunántúli Dolgozatok Természettudományi Sorozat 5:177-192.

28. JózAN, Zs. 1985b: Dél-Dunántúl kaparódarázs (Hymenoptera: Sphecoidea) faunájának alapvetése. - A Janus Pannonius Múzeum Évkönyve 29 (1984): 53-86.

29. JózAN, Zs. 1990: A Zselic méhszerú (Hymenoptera, Apoidae) faunájának alapvetése. - A Janus Pannonius Múzeum Évkönyve 34 (1989): 81-92.

30. JózAN, Zs. 1992a: A Boronka-melléki Tájvédelmi Körzet fullánkos hártyásszárnyú (Hymenoptera, Aculeata) faunájának alapvetése. - Dunántúli Dolgozatok Természettudományi Sorozat 7:163-210.

31. JóZAN, Zs. 1992b: A Zselic kaparódarázs faunájának (Hymenoptera, Aculeata) állatföldrajzi és ökofaunisztikai vizsgálata. - Somogyi Múzeumok Közleményei 9:279-292.

32. JózAn, Zs. 1995: Adatok a tervezett Duna-Dráva Nemzeti Park fullánkos hártyásszárnyú (Hymenoptera, Aculeata) faunájának ismeretéhez - Dunántúli Dolgozatok Természettudományi Sorozat 8:99-115.

33. JózAN, Zs. 1996: A Baláta környék fullánkos hártyásszárnyú faunájának (Hym. Aculeata) alapvetése. - Somogyi Múzeumok Közleményei 12: 271-297.

34. JÓZAN, Zs. 1998: A Duna-Dráva Nemzeti Park fullánkos hártyásszárnyú (Hymenoptera, Aculeata) faunája - Dunántúli Dolgozatok Természettudományi Sorozat 9:291-327.

35. JÓZAN, Zs. 2000: Külsó-Somogy méhszerú (Hymenoptera, Apoidea faunája. - Somogyi Múzeumok Közleményei 14: 309-330.

\section{The Aculeata fauna of Somogy county (Hymenoptera, Aculeata)}

\section{ZsolT JÓZAN}

The researchwork of the Aculeata fauna in Somogy county started in the 1920 's. The first data came from the southern part of Balaton region and records were done by the researchworkers of the Hungarian Natural History Museum, Bajári E., Móczár L., Móczár M. in Budapest. Part of the data was published in the Fauna Hungariae Volume XIII. and in the Hymenoptera fauna catalogue. The author started his researchwork in Somogy county in 1962. He made investigations on the fauna of Duna-Dráva National Park, Zselic, Barcs Juniper Woodland and Boronka-melléki Landscape Protection Area and Látrány Nature Conservation Area and the fauna of Outer Somogy. The results were published in several papers (JóZAN 2000).

To the present date, 992 species have been recorded in the Aculeata fauna of Somogy county (Table 1.). Column A contains the number of settlements where the species occurred and column B contains the number of papers in which the listed species were found. Crossocerus walkeri is a new species in the Hungarian Aculeata fauna.

Author's address:

Zsolt JÓZAN

H-7453 Mernye

Rákóczi út 5.

HUNGARY 